\title{
Transcription factor residence time dominates over concentration in transcription activation
}

\author{
Achim P. Popp ${ }^{1}$, Johannes Hettich ${ }^{1}$, J. Christof M. Gebhardt1,* \\ ${ }^{1}$ Institute of Biophysics, Ulm University, Albert-Einstein-Allee 11, 89081 Ulm, Germany \\ * To whom correspondence should be addressed. Email: christof.gebhardt@uni-ulm.de
}

Transcription is a vital process activated by transcription factor (TF) binding. The active gene releases a burst of transcripts before turning inactive again. While the basic course of transcription is well understood, it is unclear how binding of a TF affects the frequency, duration and size of a transcriptional burst. We systematically varied the residence time and concentration of a synthetic TF and characterized the transcription of a reporter gene by combining single molecule imaging, single molecule RNA-FISH, live transcript visualisation and analysis with a novel algorithm, Burst Inference from mRNA Distributions (BIRD). For this well-defined system, we found that TF binding solely affected burst frequency and variations in TF residence time had a stronger influence than variations in concentration. This enabled us to device a model of gene transcription, in which TF binding triggers multiple successive steps before the gene transits to the active state and actual mRNA synthesis is decoupled from TF presence. We quantified all transition times of the TF and the gene, including the TF search time and the delay between TF binding and the onset of transcription. Our quantitative measurements and analysis revealed detailed kinetic insight, which may serve as basis for a bottomup understanding of gene regulation.

\section{INTRODUCTION}

Transcription of mRNA is subject to large fluctuations. Periods of transcriptional activity of a gene, during which several mRNA molecules are produced, are followed by periods of quiescence without transcription ${ }^{1}$. This bursting behaviour has been observed for most investigated genes ${ }^{2,3}$, and in various species ranging from bacteria ${ }^{4-6}$ to yeast 7,8 and mammals ${ }^{9-12}$. Although biological processes are intrinsically stochastic due to low molecule counts and thermal forces, the large fluctuations of transcriptional bursts point to a high degree of cooperativeness within the underlying molecular mechanisms. Indeed, numerous regulatory factors of transcriptional bursting have been observed, including enhancer-promoter interactions ${ }^{13,14}$, DNA topology 4,15 , chromatin modifications and chromatin remodelers ${ }^{16-18}$, co-activators such as p300, CBP and mediator ${ }^{15}$, assembly of general transcription factors and the preinitiation complex, polymerase pausing and reinitiation ${ }^{17,19-21}$. Not least, binding of specific transcription factors (TFs) to cis regulatory sequences, usually representing the first step in the cascade of transcription activation, is associated with bursting 2,7,11,16,17,22-31.

A bursting gene can usually be described by the twostate or random-telegraph model ${ }^{32}$. There, switching of the gene between the quiescent and the active state is modelled as stochastic process with on- and off-rates, and a transcription rate describes mRNA production from the active state. First insight into the mechanism of a regulatory trait, such as enhancer action or TF binding, can be obtained by revealing which of the rates of the two-state model it influences. For TFs, it is well known that TF concentration is coupled to transcriptional activity 6,26,27,33,34. Mostly, increasing concentration was associated with an increase in burst frequency 2,16,26,27,30. An effect on burst duration ${ }^{29}$ or burst size ${ }^{2,10}$ was also reported, although care has to be taken to avoid concatenating individual bursts ${ }^{35}$.

Recently, in addition to concentration, TF residence time has been observed to affect bursting $16,22,23,28,30,31$. As with concentration, longer residence time was associated with an increase in burst frequency $16,17,28,30,31$. However, in many studies this effect was not exclusive, and an increase in burst duration 16,17,30,31 and sometimes in burst size 30,31 was also coupled to longer residence times. This mechanistic variation might trace back to the dissimilar methods of varying TF residence time. Different residence times were either achieved indirectly by studying modified TFs prevalent in certain cellular conditions 28,30,31, TF mutants with potentially different DNA binding mechanisms ${ }^{22}$, by studying different target sequences ${ }^{16}$ or by knocking out co-factors ${ }^{17}$. Overall, a clear understanding of how TF binding, in particular TF residence time and concentration, affect transcriptional bursting, and 
which of the rates of the two-state model are altered by a TF, is still missing.

Here, we decipher the role of TF residence time and concentration on bursting of a minimal reporter gene with well-defined promoter structure. We used the Auxin-Inducible Degron (AID) system ${ }^{36}$ to vary TF concentration without further genomic modification. To vary the residence time with minimal disturbances to the TF, we utilized the modular DNA binding domain of transcription-activator like effectors (TALEs) ${ }^{23}$. We quantified mRNA production and bursting parameters with single molecule Fluorescence In Situ Hybridisation (smFISH) in fixed cells, live measurements of bursting with the MS2 system, and a novel analysis tool, Bursting Inference from mRNA Distributions (BIRD). We found that both TF residence time and concentration solely affected the on-rate of the gene, leaving burst size and burst duration unaffected. Interestingly, variation of TF residence time had a stronger influence than an equal variation of concentration. We could explain our observations in terms of an extended three-state model of gene transcription, in which binding of the TF switches the gene from a quiescent to a primed state, from which multiple successive transitions have to be traversed before transcription from the active state can be initiated. Overall, our measurements and analysis reveal detailed mechanistic and kinetic information on transcription of a minimal mammalian gene, which may serve as basis for a bottom-up approach in understanding regulatory traits of gene transcription.

\section{RESULTS}

\section{Reporter cell lines to study TF residence time and concentration-dependent transcription}

We aimed at studying the influence of TF binding on gene transcription, with a focus on the leverage of TF residence time and concentration and minimal blurring by other traits such as heterogeneous TF sites. Thus, we designed a reporter gene with welldefined promoter structure including a TATA box and BRE and Inr sites based on a minimal CMV promoter (Figure $1 \mathrm{~A}$ and Supplementary information) ${ }^{37}$. This promoter exhibits minimal background expression and high activation potential. We integrated a single copy of the Tet-operator (TetO) sequence, which is not present in the human genome, to exclude cooperative effects and binding competition with endogenous TFs. The artificial gene body consisted of $630 \mathrm{bp}$, followed by 24 copies of MS2 stem loops, which enable visualizing transcription events in living cells ${ }^{9,38}$. As terminator, we chose the SV40 polyA sequence for an increased transcript level ${ }^{39}$. We inserted the reporter gene into the human osteosarcoma cell line U2-OS using the Fliplnsystem ${ }^{40}$ (Materials and Methods), as this line is well suited for single molecule fluorescence in situ hybridization (smFISH) ${ }^{23}$. We validated the integration of a single Flipln site via Southern Blot (Figure S1) and confirmed the positive Flipln reaction to insert the reporter gene via gain of Hygromycin resistance, loss of Zeocin resistance, loss of lacZ activity and different $P C R$ reactions on genomic DNA (Figure S2). After Flipln of the gene construct, we stably transfected tdMCP-tdGFP for high signal-tonoise visualization of the MS2 stem loops ${ }^{41}$.

As TFs, we designed artificial activators based on transcription activator like effectors (TALEs) ${ }^{42}$, which allow targeting any DNA sequence starting with thymine by varying their modular DNA binding domain (TALE-DBD) ${ }^{43}$. By altering the length of the TALE-DBD, TFs with different residence times can be obtained ${ }^{23}$. We therefore constructed four TALE-TFS binding to $9,13,15$ or 19 nucleotides of the TetO sequence (called T9R, T13R, T15R and T19R; Figure $1 \mathrm{~A}$, target sequences in Table S1), hypothesizing that they would also exhibit would also exhibit differences in their residence time. To exclude potential effects of TF position relative to the transcription start site, all TALE-DBDs ended at the same position. As activation domain, we used a C-terminal VP64 domain to achieve a high activation potential 44 . The TALE-TFs further possessed an N-terminal HaloTag 45 for fluorescence imaging and a nuclear localization signal (NLS) (Figure 1B and 1C).

To adjust the concentration of TFs without further genetic modulation, we utilized two copies of the mAID-tag of the Auxin-inducible-Degron (AID) system (Figure 1A) 36. After stably transfecting the enzyme responsible for ubiquitination, OsTIR1, we created four cell lines, each stably transfected with one of the TALE-TFs and selected for comparable expression levels (Figure 1B) (Materials and Methods). By comparing the intensities of TMR labelled HaloTag-TALE-TF in the nucleus with those of a HaloTag-CTCF cell line calibrated for molecular numbers as standard 46 , we determined the molar concentration of TALE-TFs within the nucleus of the four cell lines to be in the range of $100-300 \mathrm{nM}$ (Figure 1B). We could increase the expression level of TALETFs by treating cells with $200 \mu \mathrm{M}$ of the AID inhibitor Auxinole for $24 \mathrm{~h}$ (Figure 1B) 47. TALE-TFs were depleted with a half time of $28 \mathrm{~min}$ by treatment with $500 \mu \mathrm{M}$ Auxin (Figure 1C). 


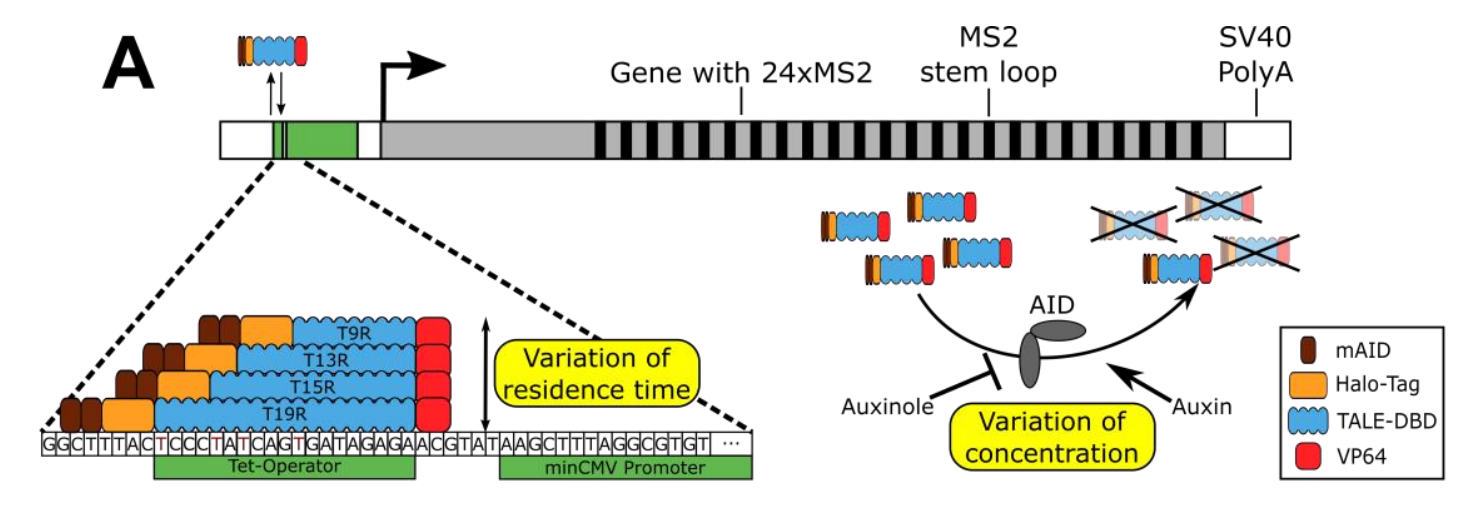

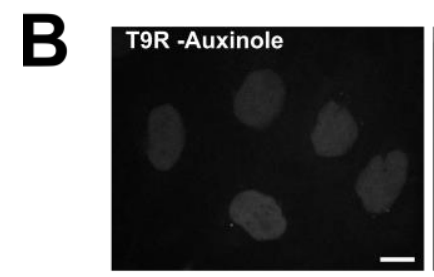

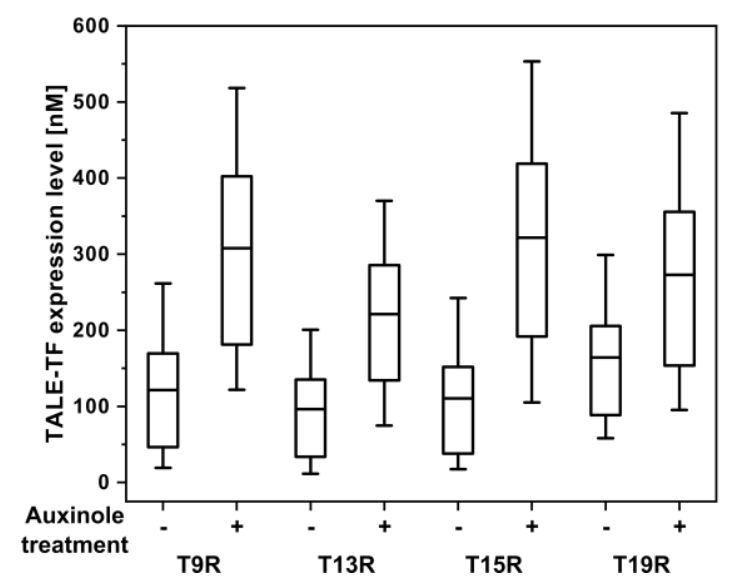

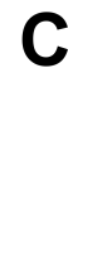
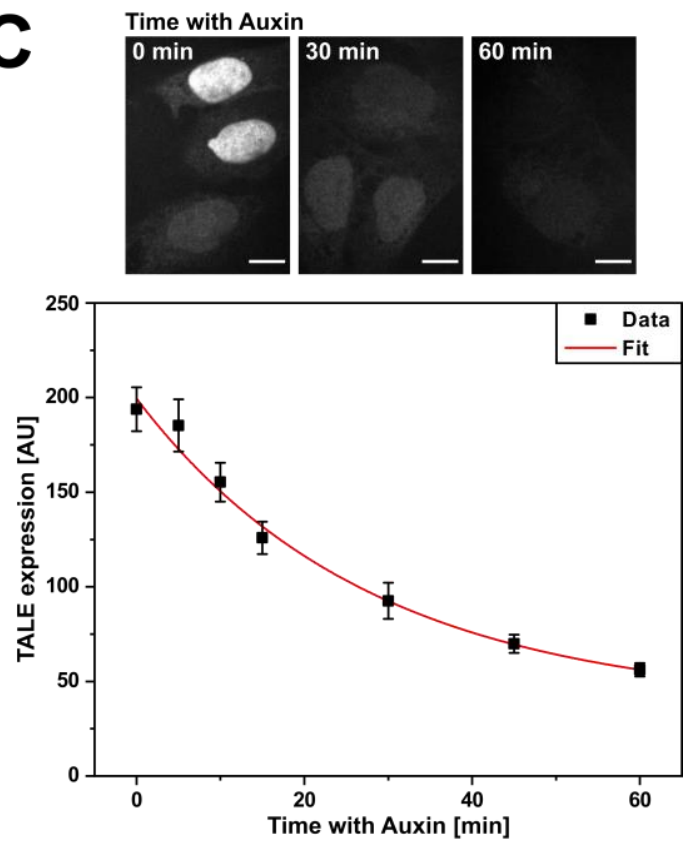

Figure 1: Design of reporter gene and strategy to vary TF residence time and concentration

A) The reporter gene consists of a TetO sequence, to which TALE-TFs are targeted, upstream of a minimal CMV-derived promoter, a 630 bp gene body, 24xMS2 repeats and a SV40 poly (A) sequence. Residence time of TALE-TFs is varied by length of the TALE DBD. Concentration of TALE-TFs is varied with the AID system and addition of either Auxinole or Auxin. B) Distribution of TALE-TF expression level with and without Auxinole treatment extracted from smFISH measurements. N: number of nuclei; $\mathrm{N}=450$ (T9R -Auxinole); $\mathrm{N}=472$ (T9R +Auxinole); N=302 (T13R -Auxinole); N=467 (T13R +Auxinole); N=476 (T15R -Auxinole); N=672 (T15R +Auxinole); $\mathrm{N}=582$ (T19R -Auxinole); $\mathrm{N}=590$ (T19R +Auxinole). Mean (line), $25^{\text {th }} / 75^{\text {th }}$ percentile (box) and $10^{\text {th }} / 90^{\text {th }}$ percentile (whiskers) define features of box plot. Inset: Fluorescence images of T9R expression without Auxinole (left) and after $16 \mathrm{~h}$ of Auxinole treatment (right). Scale bars denote $10 \mu \mathrm{m}$. C) TALE-TF expression level measured by spinning disc micoscopy and corrected for background as function of time after Auxin treatment (black squares) and mono-exponential fit (red line). ( $\mathrm{N}$ : number of nuclei; $\mathrm{N}=207$ (0min); $\mathrm{N}=207$ (5min); N=215 (10min); N=204 (15min); N=205 (30min); N=210 (45min); N=213 (60min); N=138 (background)). Error bars denote s.e.m.. Inset: Fluorescence images of T15R expression without Auxin treatment and after $30 \mathrm{~min}$ and $60 \mathrm{~min}$ of Auxin treatment. Scale bars denote $10 \mu \mathrm{m}$.

\section{TALE-TFs show differences in DNA residence time}

Before characterizing the binding kinetics of the TALE-TFs, we inserted additional reporter gene sequences in each of the TALE-TF cell lines by lentiviral gene transfer (Material and Methods). The average number of integrated copies in each cell line was 5 as determined by quantifying the activation profiles (Figure S3). To visualize single TALE-TFs, we labelled the HaloTag with the photostable organic $\mathrm{SiR}$ dye ${ }^{48}$ at low labelling densities ${ }^{49}$ and applied HILO microscopy 50 for single molecule tracking in nuclei of live cells ${ }^{23}$.

To measure the dissociation rates of TALE-TFs from chromatin, we extended the time window to long observation times using time-lapse microscopy 51 with $50 \mathrm{~ms}$ frame acquisition time and frame cycle times of $0.05 \mathrm{~s}, 1 \mathrm{~s}$ and $5 \mathrm{~s}$ (Materials and Methods). All 
constructs showed binding events ranging from less than a second up to several hundred seconds (Figure 2A, Supplementary Videos 1-3 and Figure S4). We first confirmed that overall TALE-TF binding interactions linearly increased with increasing concentration and did not exhibit binding saturation (Figure S5) ${ }^{52}$. We then accumulated the binding times of each time-lapse condition in survival time distributions (Figure 2B and Figure S4). Next, we extracted the dissociation rate spectra of TALE-TFs using genuine rate identification (GRID) (Figure 2B, $2 \mathrm{C}$ and S4) ${ }^{53}$. GRID yields the dissociation rate spectrum by inverse Laplace transformation of survival time distributions and enables correcting for photobleaching by global consideration of all timelapse conditions.
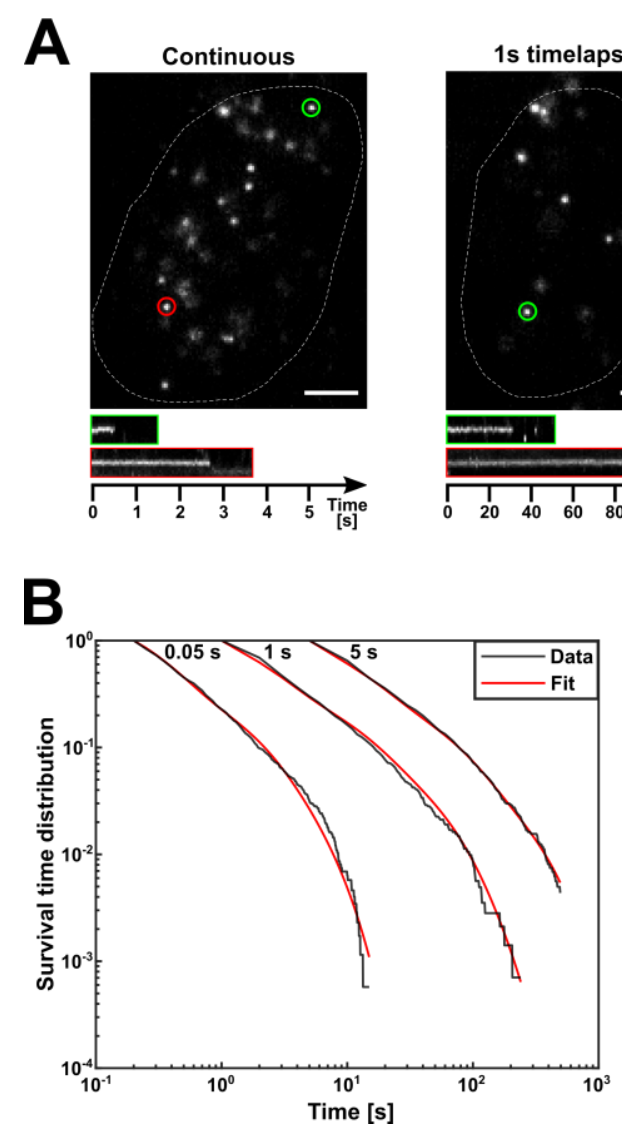
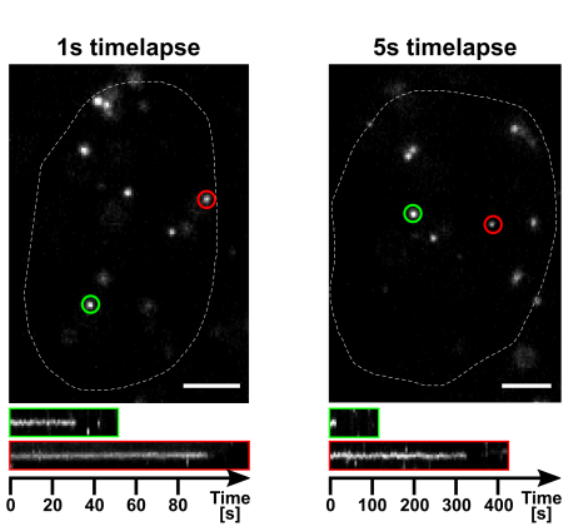

\section{D}

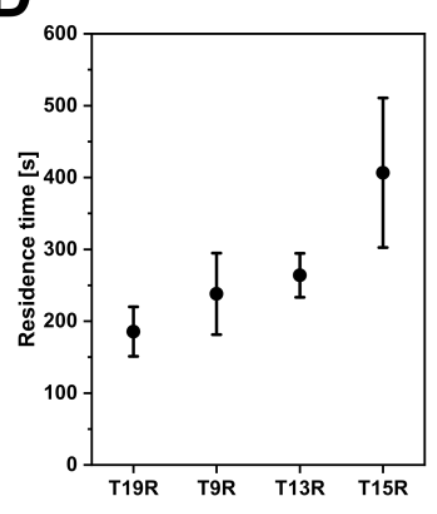

All constructs showed 6 dissociation rate clusters and equal photobleaching rates (Figure $2 \mathrm{C}$ and Table S2). The 5 dissociation rate clusters corresponding to short and intermediate binding events were similar for all constructs (Figure 2C). In contrast, the dissociation rate cluster corresponding to the longest binding events differed between all constructs (Figure 2C). Since the TALE-TFs only differ by the length of their $\mathrm{DBD}$, we reasoned that this dissociation rate cluster represents unbinding from the TetO sequence. We thus calculated the residence time from this dissociation rate cluster for all constructs and obtained an increasing series of residence times ranging from $186 \mathrm{~s}$ for $\mathrm{T} 19 \mathrm{R}$ to $407 \mathrm{~s}$ for $\mathrm{T} 15 \mathrm{R}$ (Figure 2D). We note that the variation of residence time with the length of the DBD is non-linear, similar to previous observations ${ }^{23,54}$. 
We further compared association of TALE-TFs to chromatin by calculating the number of binding events per area and time and normalized to concentration (pseudo-on-rate) (Figure S6) ${ }^{55}$. The pseudo-on-rates of all TALE-TFs were comparable. This indicates that the length of the TALE-DBD solely modifies the longest residence time of TALE-TFs.

\section{TF residence time and concentration increase transcription}

To quantify the number of mRNA molecules of the reporter gene in individual cells of the single reporter gene cell lines, we used smFISH (Figure $3 \mathrm{~A}$ and Materials and Methods) ${ }^{23,56}$. This approach also enables identifying sites of nascent mRNA

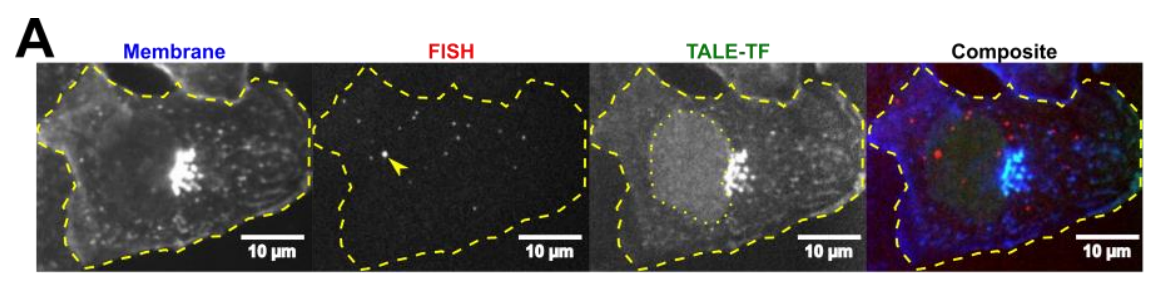

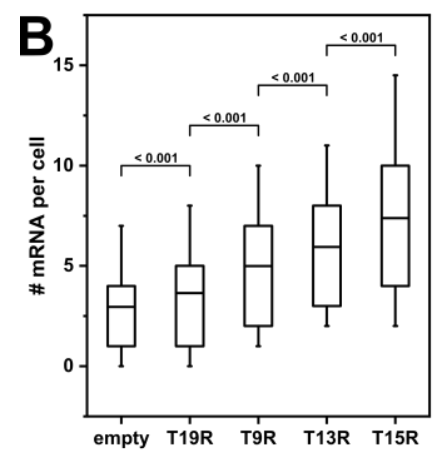

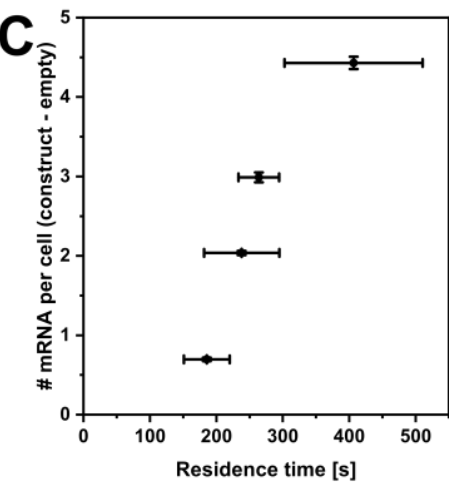

E

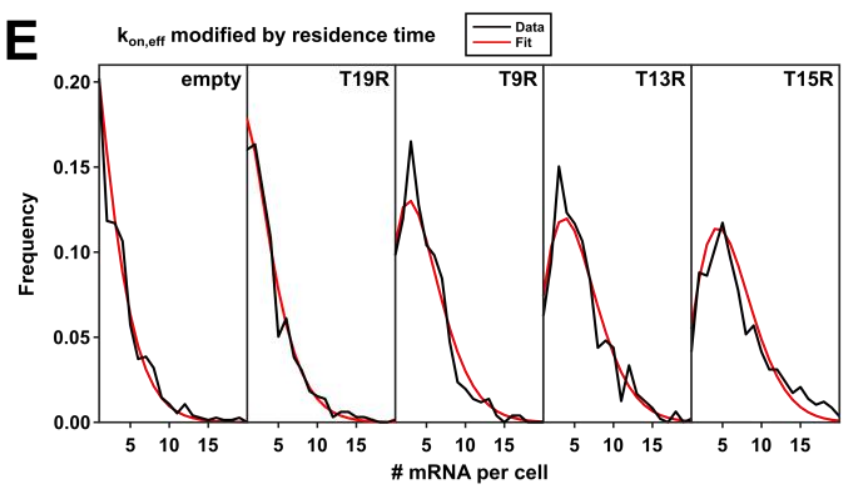

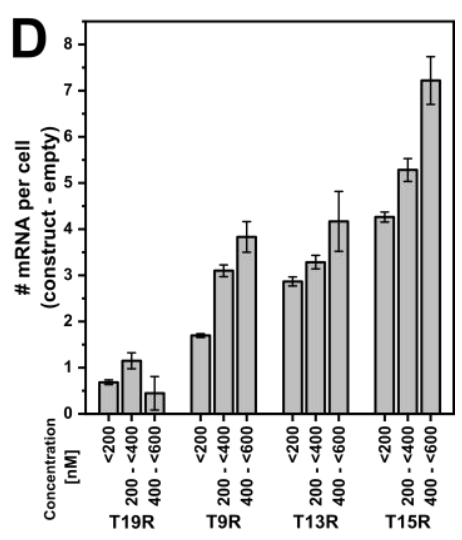

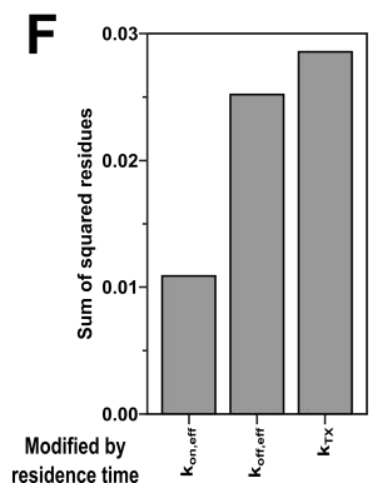

Figure 3: TF residence time and concentration stimulate transcription activation.

A) Example images of the smFISH methodology: Lectin-FITC-stained cell membrane (blue; contrast 0 3000), smFISH of specific mRNA transcripts and nascent sites of transcription (arrow) (red; z-projection; contrast 2 40), TMR-stained HaloTag-TALE-TF (green; contrast 0 500) and composite image. B) mRNA distribution in cells expressing no TALE-TF (empty) and in cells expressing the different TALE-TF with $<300 \mathrm{nM}$. Cell numbers: $N=752$ (empty); $N=888$ (T19R); $N=690$ (T9R); $N=664$ (T13R); N = 790 (T15R). Mean (line), $25^{\text {th }} / 75^{\text {th }}$ percentile (box) and $10^{\text {th }} / 90^{\text {th }}$ percentile (whiskers) define features of box plot. Significance was determined with Wilcoxon-Mann-Whitney two-sample rank test. C) Mean mRNA numbers from B) of cells expressing TALE-TF subtracted with the mean mRNA number of cells expressing no TALE-TF plotted versus TALE-TF residence time. Error bars denote s.e.m. for mean mRNA numbers and resampling error estimation for residence times. D) Mean mRNA numbers of cells expressing TALE-TF at the indicated concentration subtracted with the mean mRNA number of cells expressing no TALE-TF. Cell numbers $(<200 \mathrm{nM}, 200$ $<400 n M, 400-<600 n M)$ : T19R N=655, 375, 113; T9R $N=509$, 282, 102; T13R $N=478$, 256, 33; T15R N= $580,365,155)$. Error bars denote s.e.m.. E) mRNA distributions of TALE-TFs together with the distribution inferred by BIRD for the case where TF residence time modifies kon,eff of the gene. F) Comparison of mRNA distributions inferred by BIRD with the measured mRNA distributions of TALE-TFs by means of the lowest sum of squared residues in cases were TF residence time modifies $\mathrm{k}_{\mathrm{on}, \text { eff, }} \mathrm{k}_{\text {off, eff }}$ or $\mathrm{k}_{\mathrm{tx}}$. 
transcription, the number of nascent mRNA molecules at those sites (burst height) and the percentage of cells exhibiting active nascent transcription (burst frequency) (Materials and Methods) ${ }^{57}$. FISH probes were targeted to the SNAP gene and MS2 stem loop sequences, which are not present in the human genome and could be detected without false-positives (Figure S7). Besides mRNA, we also stained the cell membrane using Lectin-FITC and the HaloTag-TALE-TF using TMR to quantify their nuclear concentration in individual cells with U2OS Halo-CTCF C32 ${ }^{46}$ as calibrated standard (Figure $3 \mathrm{~A}$ and Figure S8).

To determine the effect of TF residence time on transcription, we compared the distributions of mRNA molecules measured by smFISH in a cell line without TALE-TF and in the four TALE-TF cell lines (Figure 3B). In these experiments, we minimized the effect of TALE-TF concentration by only considering cells with concentrations below $300 \mathrm{nM}$. All cell lines showed different mRNA levels, with mutually significant increases from empty to T19R, T9R, T13R and T15R (Figure 3B). The mean number of mRNA produced due to TALE-TF activation above the background of leaky transcription increased with TALE-TF residence time (Figure $3 C$ ). Similar to previous findings $16,22,23,28,30,31$, this suggests that TF residence time is a regulatory factor of transcription.

Next, we determined the effect of TF concentration on transcription. Therefore, we suppressed leaky AIDbased degradation ${ }^{47}$ of TALE-TFs by adding $200 \mu \mathrm{M}$ Auxinole for $24 \mathrm{~h}$ before sample preparation. We excluded an effect of Auxinole on the general transcription mechanism by comparing the mRNA distributions measured by smFISH in cells without TALE-TF in absence and presence of Auxinole (Figure S9). We then quantified the mean number of mRNA molecules in each of the TALE-TF cell lines and assigned the results to 3 concentration bins (Figure 3D). As expected from previous findings $6,26,27,33,34$, the higher the TALE-TF expression level, the higher was the number of mRNAs, again demonstrating that TF concentration is a regulatory factor of transcription.

\section{TF residence time and concentration solely modify the burst frequency}

After having shown that both TF residence time and concentration positively correlate with transcriptionactivation, we aimed at understanding whether TF residence time affected the on-rate $k_{\text {on, eff, }}$ the transcription rate $k_{t x}$ or the off-rate $k_{\text {off,gene of the }}$ gene in a two-state model of transcriptional bursting
6,11,32. We therefore developed Bursting Inference from mRNA Distributions (BIRD), an inference algorithm based on iterative fitting of RNA distributions with semi-analytical solutions to the systems of differential equations describing the gene bursting kinetics (Materials and Methods). Previously, gene kinetics was simulated with the Gillespie algorithm ${ }^{58}$ or modelled using hypergeometric series 59. We globally applied BIRD to the mRNA distributions obtained in the four TALE-TF cell lines at TF concentrations $<200 \mathrm{nM}$, while allowing only one of the rates of the two-state model to be varied at a time. Modulation of $k_{\text {on, eff }}$ by the TALE-TF residence time resulted in the best representation of our data (Figure 3E) in terms of the sum of squared residues, compared to modulating one of the other rates (Figure 3F and Figure S10).

To confirm the notion obtained from the BIRD analysis that TF residence time and concentration affect the on-rate of the reporter gene, we quantified their effect on several bursting parameters. First, we determined both frequency and height of a nascent transcription site with smFISH in each of the TALETF cell lines (Materials and Methods) ${ }^{57}$. This approach underestimates the burst frequency and overestimates the burst height, since we only considered nascent sites with four or more transcripts to minimize false positives (Materials and Methods). Nevertheless, a relative comparison of the parameters between different conditions is possible. Consistent with the BIRD analysis, both higher TF residence time and higher concentration resulted in higher burst frequency (Figure $4 \mathrm{~A}$ and $\mathrm{B}$ ), while neither TF residence time nor concentration had an effect on the burst height (Figure $4 \mathrm{~A}$ and $\mathrm{B}$ ).

The burst frequency changes if either the on-rate or the off-rate of the gene is altered, resulting in either more or longer bursts, respectively. We thus next applied the MS2 system to visualize transcription bursts in living cells and thereby directly assessed their duration (Figure 4C, Supplementary Video 4 and Materials and Methods) ${ }^{9}$. Burst durations lasted for several minutes up to several hours for the TALE-TFs with longest (T15R) and shortest (T19R) residence time, with similar average burst duration of $69 \mathrm{~min}$ for T15R and $68 \mathrm{~min}$ for T19R (Figure 4D). The cumulative frequency distributions of burst durations were well described by a mono-exponential function for both T15R and T19R, indicating a single ratedetermining step for the termination of bursts (Figure 4D). We also determined the effect of TF concentration on the burst duration, by quantifying the TMR-HaloTag labelling intensity of T15R and T19R 

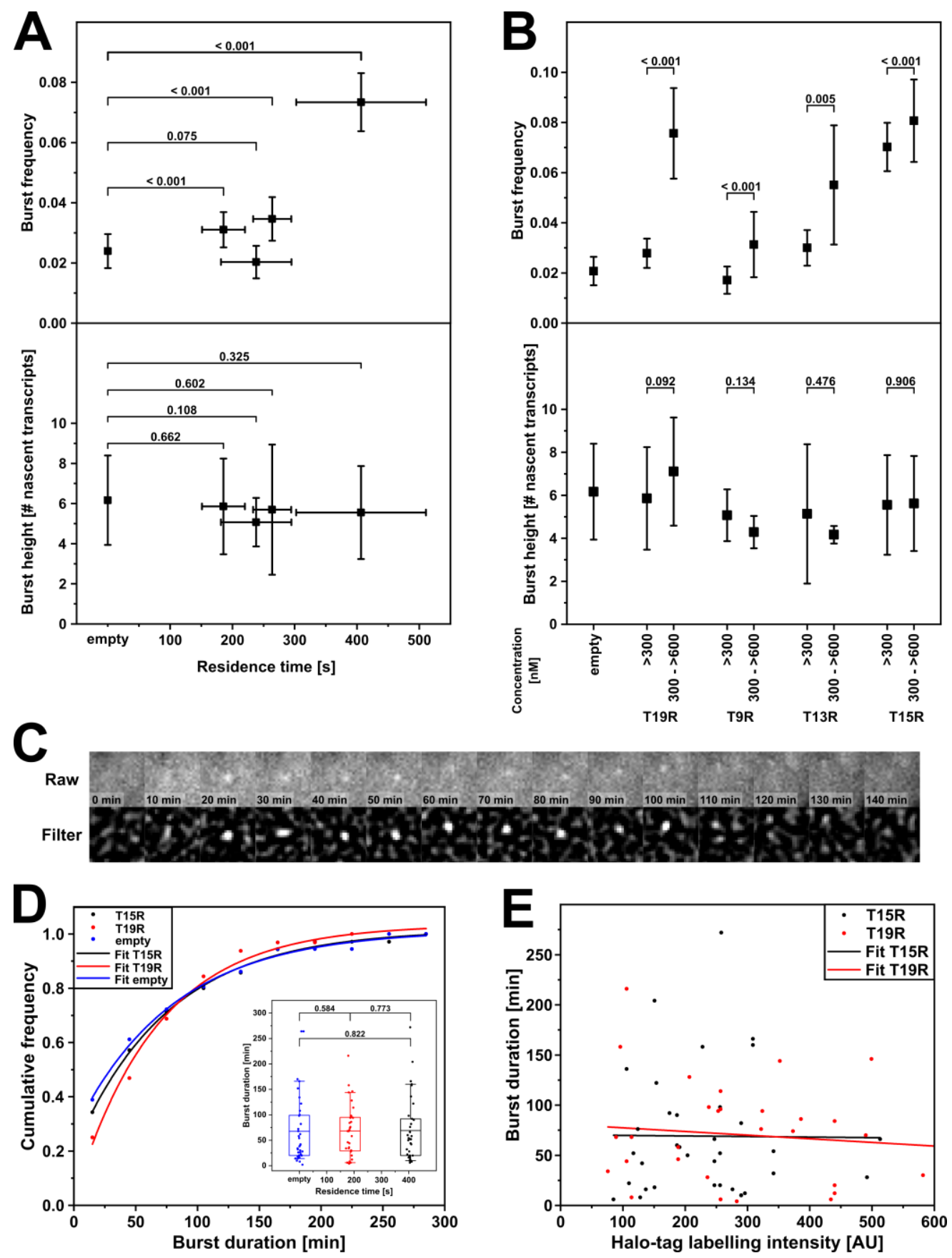

Figure 4: Effect of TF residence time and concentration on burst parameters.

A) Effect of TF residence time on burst frequency and burst height. Both parameters were determined with smFISH in cells expressing < $300 \mathrm{nM}$ TALE-TF. Number of detected transcription sites: $\mathrm{N}=19$ (empty); $\mathrm{N}=28$ (T19R); N=14 (T9R); N= 23 (T13R); N= 58 (T15R). Error bars denote sqrt(N) for burst frequencies, s.d. for burst heights and resampling error estimation for residence times. Significance was tested with unranked two sample $t$ test. B) Effect of TF concentration on burst frequency and burst height. Both parameters were determined with smFISH. Number of detected transcription sites (<300nM, $300-<600 \mathrm{nM})$ : N=19 (empty); $N=$ 28, 19 (T19R); $\mathrm{N}=14,7$ (T9R); $\mathrm{N}=23,6$ (T13R); $\mathrm{N}=58,26$ (T15R). Error bars denote sqrt(N) for burst frequencies and s.d. for burst heights. C) Example time series of a transcription site detected with the MS2 system in a living cell expressing T15R. Raw: z-projection of complete cell height; Filter: wavelet filtered zprojection. D) Cumulative frequency distributions of burst durations of cells expressing T15R (black symbols), T19R (red symbols), or no TALE-TF (empty, blue symbols). Lines represent mono-exponential fits. Number of transcription events: $\mathrm{N}=35$ (T15R), N=32 (T19R), N=36 (empty). Inset: burst duration as function of TF residence time. Mean (line), $25^{\text {th }} / 75^{\text {th }}$ percentile (box) and $10^{\text {th }} / 90^{\text {th }}$ percentile (whiskers) define features of box plot. Significance was tested with Wilcoxon-Mann-Whitney two-sample rank test. E) Scatter plot of burst duration versus TF concentration of cells expressing T15R (black symbols) or T19R (red symbols). Lines represent linear fits. (Number of transcription events: $\mathrm{N}=35$ (T15R) and $\mathrm{N}=32$ (T19R). 
(Figure 4E). As with TF residence time, burst duration was also independent of TF concentration.

We tested whether burst duration was indeed completely independent of the TF by measuring the burst duration of leaky reporter gene transcription in the cell line without TALE-TF. As predicted, the average burst duration (68 $\mathrm{min}$ ) and the cumulative frequency distribution of burst durations were comparable to the duration and distribution in presence of TF (Figure 4D). Overall, our experiments confirm the BIRD analysis and suggest that binding of a TF solely affects the on-rate $k_{\text {on,eff }}$ of the reporter gene.

\section{Long TF residence time more efficiently activates transcription than frequent TF binding}

Since both high TF residence time and high TF concentration increase transcription, the question arises whether it is more important to have long residence times or frequent association events of TFS for efficient activation of transcription. To answer this question, we calculated the fold-changes in RNA production for each difference in residence time of the four TALE-TF constructs, and for each difference in concentration of the different concentration bins (Figure 5A). A certain fold-change in TF residence time affects a larger fold-change in RNA production compared to the same fold-change in TF concentration. To better compare the severity of this effect, we normalized each RNA fold-change by the underlying parameter fold-change (Figure $5 \mathrm{~A}$ ) and found a 4 times larger effect for TF residence time.

The two-state model consisting of an off-state and an on-state of the gene, from which transcription occurs, well describes the RNA distribution of gene transcription (Figure 3E) 6,11,32. However, it is not detailed enough to include TF dissociation, in particular as the off-rate of the gene is not equal to or determined at all by binding of the TF (Figure 5B). The next simple model, a three-state model that includes a primed state from which transcription occurs upon binding of the TF and that switches to the off-state upon TF dissociation, predicts equal importance of TF residence time and TF concentration for transcription activation. To explain a dominant effect of TF residence time over TF concentration, we thus considered a multi-state model with $n$ successive states between the primed state and the on-state of the gene (Figure $5 \mathrm{~B}$ and Supplementary Information). In the case of large $n$, this model converges to the extended three-state model that predicts an exponential increase of the time necessary to switch from the off-state to the on- state with decreasing TF residence time (Supplementary Information). Indeed, the relation between $1 / k_{\text {on, eff }}$ and the dissociation rate $k_{\text {off, } T F}$ of our TALE-TFs is incompatible with a linear relation predicted by the three-state model $\left(R^{2}=0.84\right)$, but well described by the exponential behaviour of the extended three-state model $\left(R^{2}=0.94\right)$ (Figure $5 C$ ).

By combining our values for the TF residence time and the burst duration with the values for the effective on-rate, the off-rate and the transcription rate of the gene obtained by BIRD analysis of the RNA distributions, we could assign rates and values to all the transitions and parameters of the extended threestate model (Figure 5C and Supplementary Information). We found an on-rate of the TF to the gene, $\mathrm{k}_{\mathrm{on}, \mathrm{TF}}$ of $(67 \mathrm{~min})^{-1}$, burst frequencies of 0.12 , $0.15,0.22,0.25$ and 0.28 for empty, T19R, T9R, $\mathrm{T} 13 \mathrm{R}$ and T15R, a transcription rate $\mathrm{k}_{\mathrm{tx}}$ of $(28 \mathrm{~min})^{-1}$, an average burst size of 2.5 transcripts and a degradation rate $k_{d}$ of $(10 h)^{-1}$ (Supplementary Table S3). The values of burst frequency and burst size determined with BIRD are not blurred as those obtained by the smFISH measurement and approximate the true values. Interestingly, it takes $\sim 330 \mathrm{~s}$ to pass through the $\mathrm{n}$ states following TF binding, which is comparable to the TF residence time. This yields mechanistic insight into the importance of the TF residence time: While the long binding T15R on average undergoes 1 futile binding event until complete transition to the on-state of the gene and successful transcription activation, the short binding T19R already undergoes 5 futile binding events (Supplementary Information). We confirmed insufficient gene activation by short TF residence time by simulating the transcription process according to the extended three-state model using all transition rates for both $T 15 R$ and T19R (Figure 5D and Supplementary Information). T15R and T19R bind with equal frequency to the target site, but due to a lower number of futile attempts, T15R leads to a larger burst frequency than T19R.

\section{DISCUSSION}

We quantified the influence of both TF residence time and concentration on the bursting parameters of a single well-defined reporter gene. We found that both regulatory traits solely affected the rate of switching on transcription, as expected from previous reports on the role of TF binding 2,7,11,16,17,23-31. Strikingly, we found that the regulatory effect of varying TF residence time was significantly more important than the effect of varying concentration. This enabled us to 
obtain mechanistic and kinetic details about the transcription activation process beyond the common two-state or a simple three-state description of gene transcription 11,32. Rather, we found an extended three-state model was compatible with our data, in which TF binding triggers several successive transitions of the system until the gene assumes the active state and productive transcription starts. Our model is compatible with recently identified control points of transcription regulation ${ }^{19}$ and adds to
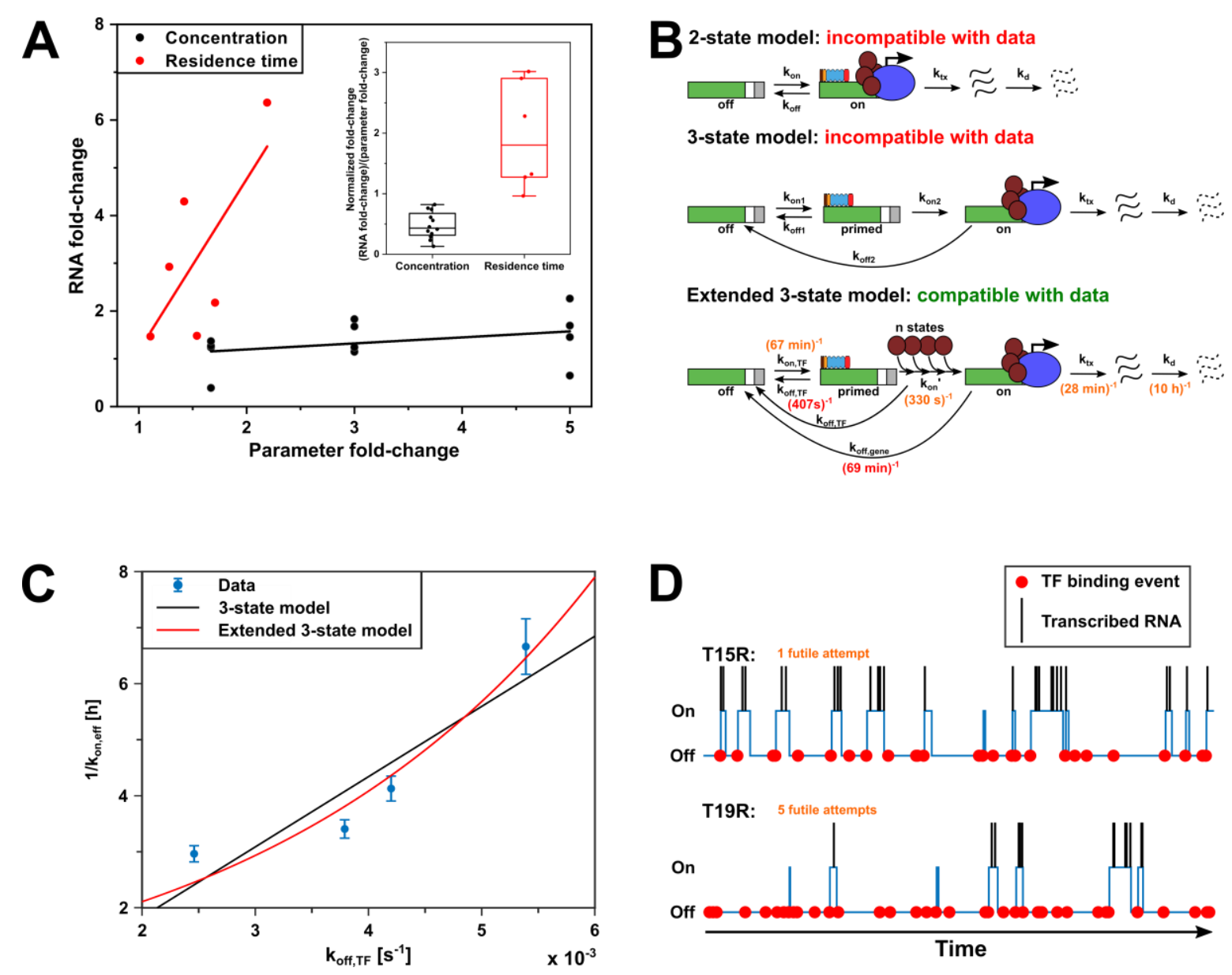

previous considerations on three-state or multi-state models 11,59-61. In our model, for which we could determine all transition rate constants, presence of the TF increases the probability of the gene to transit from the off state over a primed state to the active state. However, not just the quantity of TF interactions matters, but their quality: the probability for a successful transition to the active state is more sensitive to the TF residence time than to its concentration.

B

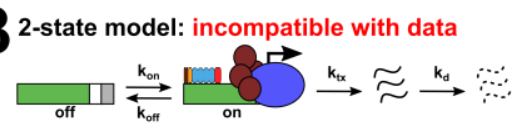

3-state model: incompatible with data

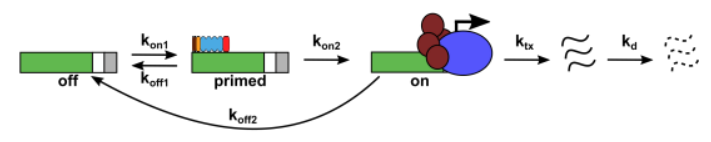

Extended 3-state model: compatible with data
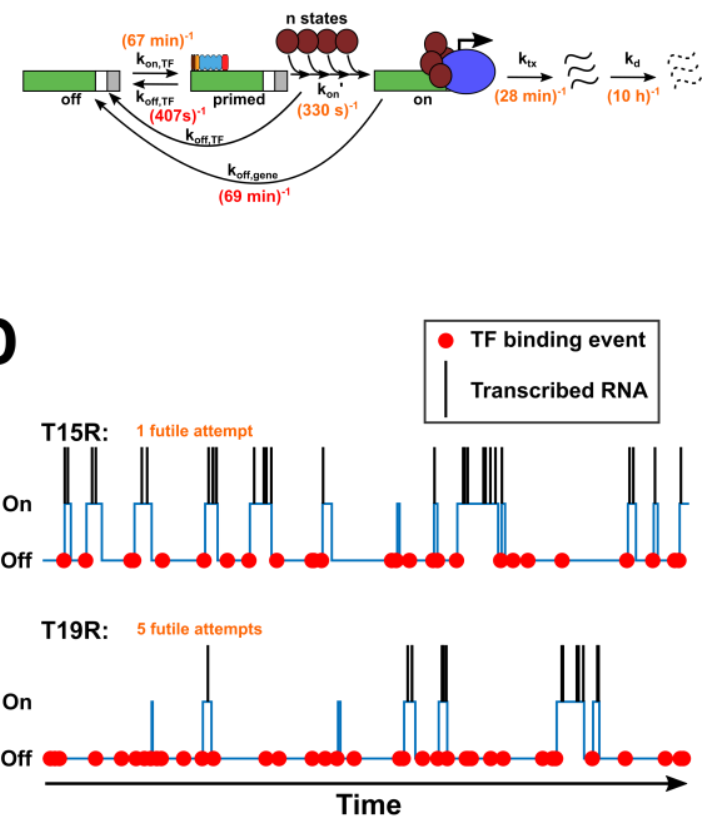

Figure 5: TF residence time dominates over concentration in transcription activation.

A) Effect of TF residence time and concentration on mRNA fold-change. mRNA fold-change was calculated from mean mRNA levels (construct - empty). Residence time fold-changes were calculated for each TALETF combination. Concentration fold-changes were calculated from the middle values of the 3 concentration bins shown in Figure 3D. Inset: RNA fold-change normalized to corresponding parameter fold-change. B) Sketches of the 2-state, 3-state and extended 3-state models of gene transcription. Rates indicated in the extended 3-state model were extracted from measurements (red font) or from BIRD and modeling (orange

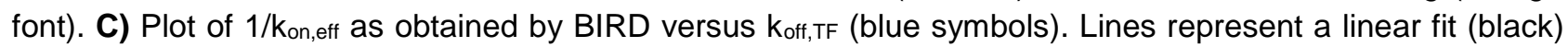
and an exponential fit (red). Error bars denote 95\% confidence interval from BIRD inference. D) Simulations of TF binding (red spheres), gene switching between off / primed and on-state (blue line) and transcriptional bursts (black lines) for T15R and T19R with transition rates of the extended 3-state model taken from measurements, BIRD and modelling (Supplementary Table S3). The average number of futile TF binding events before successful transcription is indicated.

The burst duration of 70 min and the small burst size we observed for the artificial reporter gene agree with burst parameters reported previously for endogenous mammalian genes $11,15,24,27,28,30,31,60$. At first sight, the corresponding small transcription rate with one transcript produced every 28 minutes conflicts with the fast elongation speed of RNA polymerase II of ca. $1 \mathrm{~kb} / \mathrm{min}{ }^{12,15,62-64}$. However, additional factors such 
as promoter proximal pausing of RNA polymerase II, and termination and release of mRNA influence the transcription rate. Pausing of RNA polymerase II shortly after the transcription start site is frequent and occurs with half-lives of $\sim 2$ to $30 \mathrm{~min}{ }^{65}$. For $3^{\prime}$ termination, a duration of a few minutes has been observed ${ }^{15,20}$. Together, the burst parameters we observed for the reporter gene are consistent with the current picture of mammalian gene transcription.

One of the main tasks of a TF is to recruit cofactors necessary for transcription initiation 66,67. Such recruitment processes require time and would benefit from long residence times of the TF. Indeed, for Oct4, facilitated recruitment by long binding Sox 2 has been observed ${ }^{33}$. In general, successful cofactor recruitment becomes more probable the longer the TF is associated to the promoter ${ }^{68}$. The activation domain of our TALE-TFs, VP64, directly interacts with several subunits of the mediator complex 69 . Together, possible candidates for the transitional steps between primed and active states of the gene include recruitment of the mediator complex and subsequent assembly of general transcription factors and RNA polymerase $1 /{ }^{70}$. Additional processes might include binding of histone writers and readers such as CBP, P300 and BRD4 ${ }^{71-73}$. Of note, we did not observe any major mechanistic differences between leaky transcription in absence of the TF and TFmediated gene activation. The same extended threestate model well described both cases. This suggests that the steps between the off state and the active state should be able to occur independent of the TF, albeit with much lower probability. For general TFs, a self-sufficient assembly of the pre-initiation complex is possible ${ }^{74}$. Similarly, disassembly of such a complex, or loss of histone modifications, are possible candidates for the TF-independent ratelimiting step of burst termination that we observed. As a consequence of the additional cascade of steps in the extended three-state model, an exponential relationship between the TF residence time and the number of futile binding attempts of a TF before successful transition of the gene to the active state is predicted. In contrast to T19R, T15R, which binds twice as long, efficiently activated the gene, with only one futile attempt. This is comparable to the efficiency of Mbp1p in activating an endogenous gene in yeast 7 and reflects the advantage of a long residence time for successful transcription activation.

Given the exponential dependence of successful transcription activation on TF residence time, the question arises whether residence times of hundreds of seconds as for the TALE-TFs are generally necessary for successful transcription. Residence times comparable to the TALE-TFs have been observed for other factors 31,52,75-79. However, considerably shorter residence times were reported for many TFs 22,28,33,49,80-82. In addition, low-affinity binding sites with sub-optimal TF binding might be beneficial to distinguish for TFs within the same TF family ${ }^{83}$. Thus, the cell needs ways to compensate for short residence times. Trivially, the cell could increase the TF concentration to achieve a high onrate of the gene, yet this is resource-intensive. Another possibility to increase the on-rate of the gene is to increase the number of TF target sites at the promoter ${ }^{11}$, which reduces the search time of the TF to the location ${ }^{78}$. Enhancers additionally add a prominent regulatory level to increase the burst frequency of genes ${ }^{3,13,14}$. Alternatively, the cell could increase the burst size or the burst duration once the gene is active. The burst size was found to be influenced by the nature of the activation domain 30 and the core promoter sequence ${ }^{3}$. Similarly, increasing the number of TF binding sites at the promoter was shown to increase the number of nascent transcripts ${ }^{11,30}$. Indeed, proximal promoters of many endogenous genes comprise multiple TF target sites, as do enhancer elements ${ }^{84-88}$. With an increasing number of TF sites at promoter or enhancer, the propensity of TFs to form dynamic TF condensates via their low-complexity domains increases ${ }^{89}$, as has been observed for various factors associated with transcription ${ }^{90-94}$. The size of such condensates was found to correlate with transcription output ${ }^{95}$ and GR hubs have the potential to prolong transcription bursts ${ }^{31}$. Thus, condensate formation is an elegant way to locally increase the concentration of TFs and thereby compensate for the disadvantage of short TF residence times.

We can obtain an upper limit for the time that any of the TALE-TFs present in the nucleus needs to find the single specific target site within the promoter of the reporter gene from the on-rate of the gene and the number of futile binding events. In our experiment, this target search time is $67 \mathrm{~min}$. With the concentration of TALE-TF of ca. $100 \mathrm{nM}$ in a U2-OS nucleus of approximately an ellipsoidal volume of $\pi / 6^{*} 10 \mu \mathrm{m}^{*} 10 \mu \mathrm{m}^{*} 5 \mu \mathrm{m}$, there are ca. 16,000 TALE-TF in the nucleus. Thus, the time for one TALE-TF molecule to find the target sequence is ca. $17,900 \mathrm{~h}$ (744 d). A 2.5x shorter search time of ca. 7,000 h (292 d) has been observed for TetR to find a single TetO site in U2-OS cells ${ }^{78}$. The difference might reflect differences in the search process between both factors. For one Sox2 molecule in embryonic stem cells, a search time to find any target sequence of 377 
$\mathrm{s}$ was reported, and estimated to be $31 \mathrm{~d}$ to find a single target site ${ }^{33}$, ten times shorter than for TetR. Again, different search mechanisms might account for this difference, or differences in calculating the search time from the bound fraction, residence time and number of target sites for Sox 2 versus from burst frequency and concentration for TALE-TF and the direct association measurement for TetR. Search times of ca. $100 \mathrm{~s}$ reported for p53 ${ }^{28}$ and CTCF 96 to find any target sequence face similar challenges as Sox2 to convert to the search time of one specific site. The search time of TALE-TF is significantly longer than the search time of ca. $0.1 \mathrm{~h}$ for one Lac repressor to find a single operator in E. coli (ca. $120 \mathrm{~s}$ for any TF) ${ }^{97}$, and the search time of ca. $5 \mathrm{~h}$ for one Mbp1p molecule to find a single target site in $S$. cerevisiae (ca. $50 \mathrm{~s}$ for any TF) ${ }^{7}$. The difference in search time between bacterium and yeast presumably predominantly reflects differences in chromatin organization and to some extend in genome size. The difference between yeast and human corresponds within a factor of 3 to the difference in genome size. Consequently, mammalian cells need to scale up the TF numbers compared to bacteria and yeast to compensate for the very long search time of one TF to find a single target site.

Our data suggest that the TALE-TF on average should be already dissociated once the gene is in the active state and transcription starts, since TF residence time is comparable to the transition time from the primed to the active state and the transcription rate is low. Thus, while the TALE-TF helps in transiting the gene to the active state, most RNA transcription initiation events of a burst will occur without a TALE-TF bound to the promoter. Consistently, we observed that transcription in absence of the TF followed the same kinetic model, and the burst duration was independent of the TF and its residence time. Our observations are also consistent with a recent finding that RNA polymerase II recruitment occurs only after burst initiation and is rather unregulated ${ }^{19}$, and the finding of a delay of RNA synthesis compared to GR binding of $\sim 3 \mathrm{~min}{ }^{31}$. In contrast, recent reports in yeast suggest that TF residence time is directly coupled to the burst duration, while TF concentration affects the on-rate of the gene 16,17,29. Thus, transcription in yeast apparently only occurs while the TF is bound to the promoter. This is consistent with the burst duration of a few minutes in yeast $7,16,17,29$, which is comparable to the elongation time of the gene and compatible with a few initiation events during the residence time of the TF. In comparison, typical burst durations in mammals are much longer than the TF residence time $11,15,24,27,31$. The different effects of TF residence time in mammals and yeast point to differences in the regulatory mechanisms of transcription activation in both species. For example, it seems that co-factor recruitment and assembly of the transcription machinery is more efficient in yeast compared to mammals. This might be due to differences between components of the yeast and mammalian preinitiation complexes, for example TFIID or mediator 98. It is further interesting to speculate that the ability to uncouple TF binding to and activation of the gene from RNA polymerase II recruitment enabled higher organisms to evolve enhancer elements, to which TFs predominantly bind. Our observations for the TALE-TFs predict that also an enhancer would only shortly, in the range of a few minutes, need to closely interact with a gene promoter for successful activation. Compatible with such a scenario, it has been observed that mediator condensates only transiently come into close proximity to a site of transcription while transcription continues to go on ${ }^{91}$. Further studies will be necessary to appreciate to full extend the kinetic interplay of gene activation and actual gene transcription and differences in transcription regulation between yeast and mammals.

Given the similarities in burst size, burst duration and transcription rate of the artificial reporter gene to other mammalian systems, the reporter gene with one TF target site in the proximal promoter constitutes a basis for the kinetic understanding of gene transcription. In particular, the importance of the TF residence time, the delay between TF binding and the onset of mRNA transcription, and the TF-independent transcription termination will be able to inform the kinetic behaviour of endogenous mammalian genes with more complex promoter structures.

\section{MATERIALS AND METHODS}

\section{Cloning}

\section{Gene construct}

The gene construct was synthesized by GeneArt (ThermoFisher). We integrated 24x MS2 stem loops from phage-CMV-CFP-24xMS2 (Wu et al., 2012) by Eagl-HF and Bglll restriction and ligation. Afterwards we exchanged the plasmid backbone to pcDNA5/FRT from pcDNA5/FRT/TO V $5{ }^{99}$ using Mlul$\mathrm{HF}$ and and Sphl-HF digestion. The sequence of the gene construct with inserted $24 \times M S 2$ can be found in the Supplementary Information.

For lentivirial gene transfer, we transferred the gene construct containing $24 \times \mathrm{MS} 2$ repeats into the pLenti backbone of pLenti-CMV-OsTIR1 (Section CMVOsTIR1) with restriction by Mrel-Fast and Xhol. To 
allow this, we inserted a Mrel and a Xhol restriction site into pLenti-CMV-OsTIR1 by annealing the two primers Cloning_pLenti_fw and Cloning_pLenti_rev and digestion with BamHI-HF and Clal.

\section{tdMCP-tdGFP}

To generate a tandem-GFP version of phage-ubcnls-ha-tdMCP-gfp 41, we amplified the GFP enconding sequence by $P C R$ with the primers Cloning_MCP-GFP_fw and Cloning_MCP-GFP_rev and inserted it via $\mathrm{Xbal}$ restriction and ligation. We checked for correct orientation with sequencing.

\section{CMV-OsTIR1}

We amplified OsTIR1 from pMK232 ${ }^{100}$ via PCR with the primers Cloning_OsTIR1_fw and Cloning_OsTIR1_rev and inserted it into the pLenti backbone from pLenti-CMV-rtTA3 (Addgene plasmid \#26429) by digestion with BstXI. We checked for correct orientation with sequencing.

\section{TALE-TF backbone}

We modified the pICE-Halo-VP64 plasmid from ${ }^{23}$ for this study. To hinder regulation of TALE-TF expression due to binding of the TALE-TF to their own promoter region, we removed the Tet-operators on the plasmid by Sacl-HF digestion and ligation. Then, we inserted two repeats of the mAID tag, which we amplified via PCR on pMK292 ${ }^{100}$.We therefore used for the first repeat the primers Cloning_mAID1_fw and Cloning_mAID1_rev together with HindIII-HF and Mlul-HF digestion, and for the second repeat Cloning_mAID2_fw and Cloning_mAID1_rev with only Mlul-HF digestion. We checked for correct orientation with sequencing. To ensure nuclear localization with those modifications, we inserted another nuclear localization signal C-terminally to the TALE-DBDs by annealing the two primers Cloning_NLS_fw and Cloning_NLS_rev and restriction with EcoRI-HF and Pacl.

\section{TALE-TF Golden Gate Reaction}

We assembled TALE-TF with the Golden Gate TALEN and TAL Effector Kit2.0 (Addgene kit $\# 1000000024)^{101}$ as previously described ${ }^{23}$. The designed TALE-TFs are listed with their target sequences in Table S1.

\section{Cell culture}

We performed all experiments in U2-OS based cell lines, which we cultivated at $37{ }^{\circ} \mathrm{C}$ and $5 \% \mathrm{CO}_{2}$ in DMEM supplemented with $10 \%$ FBS, $1 \%$ Glutamax, $1 \%$ nonessential amino acids, and $1 \%$ sodium pyruvate. We supplemented all antibiotics used for selection also during normal cultivation to hinder loss of the integrated sequences.

\section{Generation of cell lines}

\section{Flipln U2-OS}

We transfected U2-OS cells with linearized pFRT/lacZeo (ThermoFisher). After selection with Zeocin, we isolated colonies resulting from single cells with cloning cylinders. We screened all clones for single integration of $\mathrm{pFRT} /$ lacZeo by Southern Blot (Lofstrand Labs Limited) using a FRT site-specific probe ${ }^{18}$.

\section{FlipIn reaction}

For the Flipln reaction of the gene construct, we seeded 1.5 million Flipln U2-OS cells on a $10 \mathrm{~cm}$ dish without antibiotics. After 24 hours, we transfected the cells with $9 \mu \mathrm{g}$ pOG44 and $1 \mu \mathrm{g}$ pcDNA5/FRT-gene construct using lipofectamine 2000. After 72 hours, we started the selection with hygromycin. After selection, we isolated single colonies with cloning cylinders and screened them for positive Flipln via zeocin sensitivity, lack of $\beta$-galactosidase activity and PCR (Figure S2). To test for $\beta$-galactosidase activity, we lysed the cells with $0.5 \%$ Triton $X-100$ and incubated the lysates $24 \mathrm{~h}$ with a X-Gal containing buffer. A positive Flipln reaction prohibited formation of a blue staining. For PCR, we isolated genomic DNA as described in 102 and we validated the site specific integration via Flipln reaction with specific primers (Flipln_Test1_fw, Flipln_Test1_rev, Flipln_Test2_fw and Flipln_Test2_rev) ) (Figure S2).

\section{tdMCP-tdGFP and OsTIR1}

After Flipln of the gene construct, we stably integrated tdMCP-tdGFP and OsTIR1 into cells using a standard lentiviral production protocol (Addgene). For lentivirus production, we transfected LentiX cells with psPAX2 (Addgene \#12260), pMD2.G (Addgene \#12259), pLenti-CMV-OsTIR1 and phage-tdMCP-tdGFP. Thereafter, we exposed the cells for transfection to the harvested lentivirus. We selected transfected cells with blasticidin for OsTIR1 integration and sorted them via the GFP signal for tdMCP-tdGFP expression using FACS (BD FACSAria II).

\section{TALE-TF}

We stably transfected the TALE-TF with linearized plasmids via puromycin selection after the integration of OsTIR1 and tdMCP-tdGFP. We screened and sorted the colonies via FACS for equal expression (BD FACSAria II) of the different TALE-TF using staining with $1.25 \mu \mathrm{M}$ HaloTag-TMR ligand following the protocol of the manufacturer (Promega).

\section{Stable transfection of additional copies of gene construct}

For the single molecule tracking experiments, we generated cells with multiple copies of the gene 
construct. Therefore, we stable transfected the cell lines containing a single copy of the reporter gene, OsTIR1, tdMCP-tdGFP and one of the TALE-TF with additional copies of the gene construct via lentiviral gene transfer using a standard lentiviral production protocol (Addgene). We quantified the number of integrations by comparing the levels of transcription activation before and after lentiviral transfection of additional copies of the gene construct.

\section{AID for TALE-TF degradation}

To test TALE degradation with AID, we stained the TALE-TF with $1.25 \mu \mathrm{M}$ Halo-TMR-ligand after manufacturer protocol (Promega) in cells grown with Auxinole (Aobious) for $24 \mathrm{~h}$. Directly afterwards, we exchanged the medium to DMEM supplemented with $500 \mu \mathrm{M}$ Auxin (I2886, Sigma) to degrade TALE-TF 100. We than fixed the cells at different time points after addition of Auxin. For each time point, we determined TALE-TF expression with a SpinningDisk microscope as described in (Clauß et al., 2017). We determined the background fluorescence in cells without TALE-TF after staining with Halo-TMR-ligand.

\section{Single molecule imaging and residence time analysis \\ Preparations}

For single molecule imaging, we growed cells on glass bottom dishes (Delta T, Bioptechs). We stained the cells with 3-6 pM Halo-SiR ligand ${ }^{48}$ directly before imaging according to the protocol of the manufacturer (Promega) to obtain equal molecular densities during imaging and therefore minimize differences in tracking losses.

\section{Single molecule time-lapse imaging}

We performed single molecule imaging as described 23. To distinguish dissociation rates of TALE-TFs independently from the photobleaching rate of the SiR-dye, we performed time-lapse microscopy ${ }^{51}$. For each time-lapse condition, we introduced a different dark time between frame acquisitions of $50 \mathrm{~ms}$ exposure, resulting in frame acquisitions each $50 \mathrm{~ms}$ (continuous), $1 \mathrm{~s}$ or $5 \mathrm{~s}$ (time-lapses). To minimize differences in photobleaching rate, we controlled the laser power before each measurement to 200 $\mathrm{mW} / \mathrm{cm}^{2}$ We performed imaging up to 90 min per dish at $37^{\circ} \mathrm{C}$ in OptiMEM. We supplemented the medium for labelling and imaging with $200 \mu \mathrm{M}$ Auxinole (AOB8812, Aobious) to prevent degradation of TALETF during imaging.

\section{Tracking analysis}

We analyzed the single molecule microscopy data with Tracklt ${ }^{103}$ to obtain fluorescence survival time distributions of bound TALE-TF. We adjusted the tracking settings for a nearest neighbour tracking algorithm to minimize false-positive connections due to nearby binding events and to obtain an equal probability for tracking losses due to tracking errors and photobleaching for all time-lapse conditions. The resulting tracking settings were $\mathrm{SNR}=5$, maximal displacement of 0.6 pixels (continuous), 1.4 pixels ( 1 $\mathrm{s}$ time-lapse) and 2.0 pixels (5 s time-lapse) to separate bound from diffusing molecules and 1 gap frame for all time-lapse conditions. We used a minimal track length of 5 for continuous movies and 2 for the time-lapse conditions to minimize the effect of falsely assigned diffusing molecules to the bound population when determining the fluorescence survival time distributions of bound TALE-TF.

\section{Analysis of survival time distributions using GRID}

We determined the dissociation rate spectrum of bound TALE-TF by analysing the fluorescence survival time distributions obtained from time-lapse imaging with GRID ${ }^{53}$. In brief, GRID performs an inverse Laplace transformation of a fluorescence survival time distribution to reveal the underlying dissociation rate spectrum. To account for photobleaching, the survival time distributions of different time-lapse measurements for a TALE-TF were analysed globally.

\section{Single molecule RNA-FISH (smFISH)}

One day before sample preparations started, we seeded cells on $35 \mathrm{~mm}$ glass bottom dishes (Ibidi). We performed smFISH with additional staining of TALE-TF with 1.25 $\mu \mathrm{M}$ Halo-TMR-ligand (Promega) and of membrane with $10 \mu \mathrm{g} / \mathrm{ml}$ Lectin-FITC (SigmaAldrich) after a modified Stellaris RNA-FISH protocol (Biosearch Technologies) as described in ${ }^{23}$. In brief, we hybridized fluorescently labelled probes targeting SNAP-tag and MS2 repeats to their target mRNA for $16 \mathrm{~h}$ to increase the signal of the target RNAs. This was followed by a total of 5 washing steps with a final washing time of $16 \mathrm{~h}$ to lower the background signal. We ordered the probes as a Stellaris Custom Probe set (Biosearch Technologies) and they are listed in Table S4. We analysed the smFISH data with the Matlab toolbox FISH-quant using the membrane staining to draw the cell outlines ${ }^{57}$. Transcription sites were detected as brightest spot in the nucleus with a minimum of 4 transcripts to minimize false-positive detections and with a cut-off of the $5 \%$ brightest spots. We used the Halo-TMR staining to quantify nuclear TALE-TF concentrations, with U2-OS HaloCTCF C32 ${ }^{46}$ as calibrated reference (Figure S8 and Formula S1).

To achieve a higher expression of the TALE-TF in the same cell lines, we inhibited leaky degradation via the 
AID system ${ }^{47}$ by supplementing medium with $200 \mu \mathrm{M}$ Auxinole (AOB8812, Aobious) $24 \mathrm{~h}$ before sample preparation.

\section{MS2 measurements}

For life cell transcription measurements, we seeded cells on 2-well dishes (Ibidi). To simultaneously quantifiy TALE-TF expression levels, we stained the cells directly before the measurement with $1.25 \mu \mathrm{M}$ Halo-TMR-ligand according to the protocol of the manufacturer (Promega). We supplemented the medium for labelling and imaging with $200 \mu \mathrm{M}$ Auxinole (Aobious) to prevent leaky degradation of TALE-TF during imaging. We omitted Halo-tag labelling and incubation with Auxinole for the cell line containing no TALE-TF.

We performed imaging with a spinning disk microscope ${ }^{23}$ equipped with an additional cultivation chamber for temperature and $\mathrm{CO}_{2}$ control (Pecon). We imaged the Cells were in phenolred-free DMEM at $37^{\circ} \mathrm{C}$ and $5 \% \mathrm{CO}_{2}$ by taking z-stacks of the entire nuclear volume (typically $12.5 \mu \mathrm{m}$ ) with a step size of $500 \mathrm{~nm}$ and exposure times of $200 \mathrm{~ms}$ every $2 \mathrm{~min}$.

We analysed the duration of transcriptional bursts with Tracklt. We adjusted the SNR for each cell individually depending on the brightness of the burst and the background signal. For tracking, we used a maximal displacement of $2 \mu \mathrm{m}$, a minimal track length of 2 and 2 gap frames to minimize premature loss of tracks.

\section{ACKNOWLEDGMENTS}

We thank Astrid Bellan-Koch for her help with cloning. phage-CMV-CFP-24xMS2 (Addgene plasmid \#40651) and phage-ubc-nls-ha-tdMCP-gfp (Addgene plasmid \#40649) were gifts from Robert Singer (Albert Einstein College of Medicine, New York, USA). pcDNA5/FRT/TO V5 (Addgene plasmid \#19445) was a gift from Harm Kampinga (University of Groningen, Groningen, The Netherlands). pMK232 (CMV-OsTIR1-PURO; Addgene plasmid \#72834) and pMK292 (mAID-mCherry2-NeoR; Addgene plasmid \#72830) were gifts from Masato Kanemakie (National Institute of Genetics, Mishima, Japan). pLenti-CMV-rtTA3 (Addgene plasmid \#26429) was a gift from Eric Campeau (University of Massachusetts Medical School, Worcester, USA). pOG44 was kindly provided by David Suter (Ecole Polytechnique Fédérale de Lausanne, Lausanne, Switzerland). Halo-SiR ligand was kindly provided by K. Johnsson (Max Planck Institute for Medical Research, Heidelberg, Germany). We thank the Core Facility FACS of Ulm University for their help with cell sorting, with special thanks to Dr. Simona Ursu and Daniela
Froelich. The authors thank the Ulm University Center for Translational Imaging MoMAN for its support.

\section{AUTHOR CONTRIBUTIONS}

J.C.M.G. conceived the study; A.P.P. and J.C.M.G. designed experimental approaches; A.P.P. designed and performed cloning and cell line generation; A.P.P. performed experiments; A.P.P. and J.H. analysed data; J.H. devised the BIRD algorithm; J.H. and J.C.M.G modelled data; A.P.P. and J.C.M.G wrote the manuscript with input from all authors.

\section{FUNDING}

The work was funded by the European Research Council (ERC) under the European Union's Horizon 2020 Research and Innovation Program (No. 637987 ChromArch to J.C.M.G.), the German Research Foundation (SPP 2202 GE 2631/2-1 to J.C.M.G.) and the Carl Zeiss Foundation (to A.P.P.). J.C.M.G. acknowledges support by the Collaborative Research Centre 1279 (DFG 316249678).

\section{CONFLICT OF INTEREST STATEMENT}

The authors declare no conflict of interest.

\section{DATA AVAILABILTY}

Data supporting the findings of this manuscript will be available from the corresponding author after publication upon reasonable request. All raw single particle tracking data, mRNA distributions, burst durations and AID degradation data will be freely available after publication.

\section{CODE AVAILABILITY}

The BIRD algorithm is freely available. A MatLab version of BIRD is available at https://gitlab.com/GebhardtLab/BIRD.

\section{REFERENCES}

1. Rodriguez, J. \& Larson, D. R. Transcription in Living Cells: Molecular Mechanisms of Bursting. Annu. Rev. Biochem. 89, null (2020).

2. Dar, R. D. et al. Transcriptional burst frequency and burst size are equally modulated across the human genome. Proc. Natl. Acad. Sci. 109, 17454-17459 (2012).

3. Larsson, A. J. M. et al. Genomic encoding of transcriptional burst kinetics. Nature 565, 251254 (2019). 
4. Chong, S., Chen, C., Ge, H. \& Xie, X. S. Mechanism of Transcriptional Bursting in Bacteria. Cell 158, 314-326 (2014).

5. Golding, I., Paulsson, J., Zawilski, S. M. \& Cox, E. C. Real-Time Kinetics of Gene Activity in Individual Bacteria. Cell 123, 1025-1036 (2005).

6. So, L. et al. General properties of transcriptional time series in Escherichia coli. Nat. Genet. 43, 554-560 (2011).

7. Larson, D. R., Zenklusen, D., Wu, B., Chao, J. A. \& Singer, R. H. Real-Time Observation of Transcription Initiation and Elongation on an Endogenous Yeast Gene. Science 332, 475478 (2011).

8. Zenklusen, D., Larson, D. R. \& Singer, R. H. Single-RNA counting reveals alternative modes of gene expression in yeast. Nat. Struct. Mol. Biol. 15, 1263-1271 (2008).

9. Chubb, J. R., Trcek, T., Shenoy, S. M. \& Singer, R. H. Transcriptional Pulsing of a Developmental Gene. Curr. Biol. 16, 10181025 (2006).

10. Raj, A., Peskin, C. S., Tranchina, D., Vargas, D. Y. \& Tyagi, S. Stochastic mRNA Synthesis in Mammalian Cells. PLoS Biol 4, e309 (2006).

11. Suter, D. M. et al. Mammalian Genes Are Transcribed with Widely Different Bursting Kinetics. Science 332, 472-474 (2011).

12. Yunger, S., Rosenfeld, L., Garini, Y. \& ShavTal, Y. Single-allele analysis of transcription kinetics in living mammalian cells. Nat. Methods 7, 631-633 (2010).

13. Bartman, C. R., Hsu, S. C., Hsiung, C. C.-S., Raj, A. \& Blobel, G. A. Enhancer Regulation of Transcriptional Bursting Parameters Revealed by Forced Chromatin Looping. Mol. Cell 62, 237-247 (2016).

14. Fukaya, T., Lim, B. \& Levine, M. Enhancer Control of Transcriptional Bursting. Cell 166, 358-368 (2016).

15. Tantale, K. et al. A single-molecule view of transcription reveals convoys of RNA polymerases and multi-scale bursting. Nat. Commun. 7, 12248 (2016).

16. Donovan, B. T. et al. Live-cell imaging reveals the interplay between transcription factors, nucleosomes, and bursting. EMBO J. 38, e100809 (2019).
17. Mehta, G. D. et al. Single-Molecule Analysis Reveals Linked Cycles of RSC Chromatin Remodeling and Ace1p Transcription Factor Binding in Yeast. Mol. Cell 72, 875-887.e9 (2018).

18. Nicolas, D., Zoller, B., Suter, D. M. \& Naef, F. Modulation of transcriptional burst frequency by histone acetylation. Proc. Natl. Acad. Sci. 115, 7153-7158 (2018).

19. Bartman, C. R. et al. Transcriptional Burst Initiation and Polymerase Pause Release Are Key Control Points of Transcriptional Regulation. Mol. Cell 73, 519-532.e4 (2019).

20. Tantale, K. et al. Stochastic pausing at latent HIV-1 promoters generates transcriptional bursting. bioRxiv 2020.08.25.265413 (2020) doi:10.1101/2020.08.25.265413.

21. van den Berg, A. A. \& Depken, M. Crowdinginduced transcriptional bursts dictate polymerase and nucleosome density profiles along genes. Nucleic Acids Res. 45, 76237632 (2017).

22. Callegari, A. et al. Single-molecule dynamics and genome-wide transcriptomics reveal that NF-kB (p65)-DNA binding times can be decoupled from transcriptional activation. PLOS Genet. 15, e1007891 (2019).

23. Clauß, K. et al. DNA residence time is a regulatory factor of transcription repression. Nucleic Acids Res. 45, 11121-11130 (2017).

24. Fritzsch, C. et al. Estrogen-dependent control and cell-to-cell variability of transcriptional bursting. Mol. Syst. Biol. 14, e7678 (2018).

25. Kalo, A. et al. Cellular Levels of Signaling Factors Are Sensed by $\beta$-actin Alleles to Modulate Transcriptional Pulse Intensity. Cell Rep. 11, 419-432 (2015).

26. Ko, M. S., Nakauchi, H. \& Takahashi, N. The dose dependence of glucocorticoid-inducible gene expression results from changes in the number of transcriptionally active templates. EMBO J. 9, 2835-2842 (1990).

27. Larson, D. R. et al. Direct observation of frequency modulated transcription in single cells using light activation. eLife 2, (2013).

28. Loffreda, A. et al. Live-cell p53 single-molecule binding is modulated by $\mathrm{C}$-terminal acetylation and correlates with transcriptional activity. Nat. Commun. 8, 1-12 (2017). 
29. Rullan, M., Benzinger, D., Schmidt, G. W., Milias-Argeitis, A. \& Khammash, M. An Optogenetic Platform for Real-Time, Single-Cell Interrogation of Stochastic Transcriptional Regulation. Mol. Cell 70, 745-756.e6 (2018).

30. Senecal, A. et al. Transcription Factors Modulate c-Fos Transcriptional Bursts. Cell Rep. 8, 75-83 (2014).

31. Stavreva, D. A. et al. Transcriptional Bursting and Co-bursting Regulation by Steroid Hormone Release Pattern and Transcription Factor Mobility. Mol. Cell 75, 1161-1177.e11 (2019).

32. Peccoud, J. \& Ycart, B. Markovian Modeling of Gene-Product Synthesis. Theor. Popul. Biol. 48, 222-234 (1995).

33. Chen, J. et al. Single-Molecule Dynamics of Enhanceosome Assembly in Embryonic Stem Cells. Cell 156, 1274-1285 (2014).

34. Lee, R. E. C., Walker, S. R., Savery, K., Frank, D. A. \& Gaudet, S. Fold Change of Nuclear NFKB Determines TNF-Induced Transcription in Single Cells. Mol. Cell 53, 867-879 (2014).

35. Wang, Y., Qi, J., Shao, J. \& Tang, X.-Q. Signaling Mechanism of Transcriptional Bursting: A Technical Resolution-Independent Study. Biology 9, 339 (2020).

36. Nishimura, K., Fukagawa, T., Takisawa, H., Kakimoto, T. \& Kanemaki, M. An auxin-based degron system for the rapid depletion of proteins in nonplant cells. Nat. Methods 6, 917922 (2009).

37. Loew, R., Heinz, N., Hampf, M., Bujard, H. \& Gossen, M. Improved Tet-responsive promoters with minimized background expression. BMC Biotechnol. 10, 81 (2010).

38. Bertrand, E. et al. Localization of ASH1 mRNA Particles in Living Yeast. Mol. Cell 2, 437-445 (1998).

39. Salem, T. Z. et al. The Influence of SV40 polyA on Gene Expression of Baculovirus Expression Vector Systems. PLoS ONE 10, (2015).

40. O'Gorman, S., Fox, D. T. \& Wahl, G. M. Recombinase-Mediated Gene Activation and Site-Specific Integration in Mammalian Cells. Science 251, 1351-1355 (1991).

41. Wu, B., Chao, J. A. \& Singer, R. H. Fluorescence Fluctuation Spectroscopy Enables Quantitative Imaging of Single mRNAs in Living Cells. Biophys. J. 102, 2936-2944 (2012).

42. Boch, J. \& Bonas, U. Xanthomonas AvrBs3 Family-Type III Effectors: Discovery and Function. Annu. Rev. Phytopathol. 48, 419-436 (2010).

43. Boch, J. et al. Breaking the Code of DNA Binding Specificity of TAL-Type III Effectors. Science 326, 1509-1512 (2009).

44. Beerli, R. R., Segal, D. J., Dreier, B. \& Barbas, C. F. Toward controlling gene expression at will: Specific regulation of the erbB-2/HER-2 promoter by using polydactyl zinc finger proteins constructed from modular building blocks. Proc. Natl. Acad. Sci. 95, 14628-14633 (1998).

45. Los, G. V. et al. HaloTag: A Novel Protein Labeling Technology for Cell Imaging and Protein Analysis. ACS Chem. Biol. 3, 373-382 (2008).

46. Cattoglio, C. et al. Determining cellular CTCF and cohesin abundances to constrain 3D genome models. eLife 8, e40164 (2019).

47. Yesbolatova, A., Natsume, T., Hayashi, K. \& Kanemaki, M. T. Generation of conditional auxin-inducible degron (AID) cells and tight control of degron-fused proteins using the degradation inhibitor auxinole. Methods 164165, 73-80 (2019).

48. Lukinavičius, G. et al. A near-infrared fluorophore for live-cell super-resolution microscopy of cellular proteins. Nat. Chem. 5, 132-139 (2013).

49. Mazza, D., Abernathy, A., Golob, N., Morisaki, T. \& McNally, J. G. A benchmark for chromatin binding measurements in live cells. Nucleic Acids Res. 40, e119-e119 (2012).

50. Tokunaga, M., Imamoto, N. \& Sakata-Sogawa, K. Highly inclined thin illumination enables clear single-molecule imaging in cells. Nat. Methods 5, 159-161 (2008).

51. Gebhardt, J. C. M. et al. Single-molecule imaging of transcription factor binding to DNA in live mammalian cells. Nat. Methods 10, 421426 (2013).

52. Reisser, M. et al. Single-molecule imaging correlates decreasing nuclear volume with increasing TF-chromatin associations during zebrafish development. Nat. Commun. 9, 1-11 (2018). 
53. Reisser, M. et al. Inferring quantity and qualities of superimposed reaction rates from single molecule survival time distributions. Sci. Rep. 10, 1-13 (2020).

54. Geiger-Schuller, K., Mitra, J., Ha, T. \& Barrick, D. Functional instability allows access to DNA in longer transcription Activator-Like effector (TALE) arrays. eLife 8, e38298 (2019).

55. Raccaud, M. et al. Mitotic chromosome binding predicts transcription factor properties in interphase. Nat. Commun. 10, 487 (2019).

56. Raj, A., van den Bogaard, P., Rifkin, S. A., van Oudenaarden, A. \& Tyagi, S. Imaging individual mRNA molecules using multiple singly labeled probes. Nat. Methods 5, 877-879 (2008).

57. Mueller, F. et al. FISH-quant: automatic counting of transcripts in 3D FISH images. Nat. Methods 10, 277-278 (2013).

58. Gorin, G., Wang, M., Golding, I. \& Xu, H. Stochastic simulation and statistical inference platform for visualization and estimation of transcriptional kinetics. PLOS ONE 15, e0230736 (2020).

59. Ham, L., Schnoerr, D., Brackston, R. D. \& Stumpf, M. P. H. Exactly solvable models of stochastic gene expression. J. Chem. Phys. 152, 144106 (2020).

60. Corrigan, A. M., Tunnacliffe, E., Cannon, D. \& Chubb, J. R. A continuum model of transcriptional bursting. eLife 5, e13051 (2016).

61. Zoller, B., Nicolas, D., Molina, N. \& Naef, F. Structure of silent transcription intervals and noise characteristics of mammalian genes. Mol. Syst. Biol. 11, 823 (2015).

62. Boireau, S. et al. The transcriptional cycle of HIV-1 in real-time and live cells. J. Cell Biol. 179, 291-304 (2007).

63. Femino, A. M., Fay, F. S., Fogarty, K. \& Singer, R. H. Visualization of Single RNA Transcripts in Situ. Science 280, 585-590 (1998).

64. Maiuri, P. et al. Fast transcription rates of RNA polymerase II in human cells. EMBO Rep. 12, 1280-1285 (2011).

65. Core, L. \& Adelman, K. Promoter-proximal pausing of RNA polymerase II: a nexus of gene regulation. Genes Dev. 33, 960-982 (2019).

66. Chen, K. \& Rajewsky, N. The evolution of gene regulation by transcription factors and microRNAs. Nat. Rev. Genet. 8, 93-103 (2007).
67. Lemon, B. \& Tjian, R. Orchestrated response: a symphony of transcription factors for gene control. Genes Dev. 14, 2551-2569 (2000).

68. Hettich, J. \& Gebhardt, J. C. M. Transcription factor target site search and gene regulation in a background of unspecific binding sites. J. Theor. Biol. 454, 91-101 (2018).

69. Poss, Z. C., Ebmeier, C. C. \& Taatjes, D. J. The Mediator complex and transcription regulation. Crit. Rev. Biochem. Mol. Biol. 48, 575-608 (2013).

70. Soutourina, J. Transcription regulation by the Mediator complex. Nat. Rev. Mol. Cell Biol. 19, 262-274 (2018).

71. Bártová, E., Krejčí, J., Harničarová, A., Galiová, G. \& Kozubek, S. Histone Modifications and Nuclear Architecture: A Review. J. Histochem. Cytochem. 56, 711-721 (2008).

72. Li, B., Carey, M. \& Workman, J. L. The Role of Chromatin during Transcription. Cell 128, 707719 (2007).

73. Wu, S.-Y. \& Chiang, C.-M. The Double Bromodomain-containing Chromatin Adaptor Brd4 and Transcriptional Regulation. J. Biol. Chem. 282, 13141-13145 (2007).

74. Cramer, P. Organization and regulation of gene transcription. Nature 573, 45-54 (2019).

75. Chen, D., Hinkley, C. S., Henry, R. W. \& Huang, S. TBP Dynamics in Living Human Cells: Constitutive Association of TBP with Mitotic Chromosomes. Mol. Biol. Cell 13, 276-284 (2002).

76. Hammar, P. et al. Direct measurement of transcription factor dissociation excludes a simple operator occupancy model for gene regulation. Nat. Genet. 46, 405-408 (2014).

77. Hipp, L. et al. Single-molecule imaging of the transcription factor SRF reveals prolonged chromatin-binding kinetics upon cell stimulation. Proc. Natl. Acad. Sci. 116, 880-889 (2019).

78. Normanno, D. et al. Probing the target search of DNA-binding proteins in mammalian cells using TetR as model searcher. Nat. Commun. 6, 1-10 (2015).

79. Teves, S. S. et al. A stable mode of bookmarking by TBP recruits RNA polymerase II to mitotic chromosomes. elife 7, e35621 (2018). 
80. Morisaki, T., Müller, W. G., Golob, N., Mazza, D. \& McNally, J. G. Single molecule analysis of transcription factor binding at transcription sites in live cells. Nat. Commun. 5, 4456 (2014).

81. Speil, J. et al. Activated STAT1 Transcription Factors Conduct Distinct Saltatory Movements in the Cell Nucleus. Biophys. J. 101, 2592-2600 (2011).

82. Sugo, N. et al. Single-Molecule Imaging Reveals Dynamics of CREB Transcription Factor Bound to Its Target Sequence. Sci. Rep. 5, 10662 (2015).

83. Kribelbauer, J. F., Rastogi, C., Bussemaker, H. J. \& Mann, R. S. Low-Affinity Binding Sites and the Transcription Factor Specificity Paradox in Eukaryotes. Annu. Rev. Cell Dev. Biol. 35, 357379 (2019).

84. Haberle, V. \& Stark, A. Eukaryotic core promoters and the functional basis of transcription initiation. Nat. Rev. Mol. Cell Biol. 19, 621-637 (2018).

85. Morgunova, E. \& Taipale, J. Structural perspective of cooperative transcription factor binding. Curr. Opin. Struct. Biol. 47, 1-8 (2017).

86. Reiter, F., Wienerroither, S. \& Stark, A. Combinatorial function of transcription factors and cofactors. Curr. Opin. Genet. Dev. 43, 7381 (2017).

87. Scruggs, B. S. et al. Bidirectional Transcription Arises from Two Distinct Hubs of Transcription Factor Binding and Active Chromatin. Mol. Cell 58, 1101-1112 (2015).

88. Taher, L., Smith, R. P., Kim, M. J., Ahituv, N. \& Ovcharenko, I. Sequence signatures extracted from proximal promoters can be used to predict distal enhancers. Genome Biol. 14, R117 (2013).

89. Chong, S. et al. Imaging dynamic and selective low-complexity domain interactions that control gene transcription. Science 361, (2018).

90. Boija, A. et al. Transcription Factors Activate Genes through the Phase-Separation Capacity of Their Activation Domains. Cell 175, 18421855.e16 (2018).

91. Cho, W.-K. et al. Mediator and RNA polymerase II clusters associate in transcription-dependent condensates. Science 361, 412-415 (2018).
92. Cisse, I. I. et al. Real-Time Dynamics of RNA Polymerase II Clustering in Live Human Cells. Science 341, 664-667 (2013).

93. Guo, Y. E. et al. Pol II phosphorylation regulates a switch between transcriptional and splicing condensates. Nature 572, 543-548 (2019).

94. Sabari, B. R. et al. Coactivator condensation at super-enhancers links phase separation and gene control. Science 361, (2018).

95. Cho, W.-K. et al. RNA Polymerase II cluster dynamics predict mRNA output in living cells. eLife 5, e13617 (2016).

96. Hansen, A. S., Amitai, A., Cattoglio, C., Tjian, R. \& Darzacq, X. Guided nuclear exploration increases CTCF target search efficiency. Nat. Chem. Biol. 16, 257-266 (2020).

97. Elf, J., Li, G.-W. \& Xie, X. S. Probing Transcription Factor Dynamics at the SingleMolecule Level in a Living Cell. Science 316, 1191-1194 (2007).

98. Schier, A. C. \& Taatjes, D. J. Structure and mechanism of the RNA polymerase II transcription machinery. Genes Dev. 34, 465488 (2020).

99. Hageman, J. \& Kampinga, H. H. Computational analysis of the human HSPH/HSPA/DNAJ family and cloning of a human HSPH/HSPA/DNAJ expression library. Cell Stress Chaperones 14, 1-21 (2009).

100. Natsume, T., Kiyomitsu, T., Saga, Y. \& Kanemaki, M. T. Rapid Protein Depletion in Human Cells by Auxin-Inducible Degron Tagging with Short Homology Donors. Cell Rep. 15, 210-218 (2016).

101. Cermak, T. et al. Efficient design and assembly of custom TALEN and other TAL effector-based constructs for DNA targeting. Nucleic Acids Res. gkr218 (2011) doi:10.1093/nar/gkr218.

102. Truett, G. E. et al. Preparation of PCR-quality mouse genomic DNA with hot sodium hydroxide and tris (HotSHOT). BioTechniques 29, 52, 54 (2000).

103. Kuhn, T., Hettich, J. \& Gebhardt, J. C. M. Single molecule tracking and analysis framework including theory-predicted parameter settings. bioRxiv 2020.11.25.398271 